\title{
Potential of silver-kaolin in gelatin composite films as active food packaging materials
}

\begin{abstract}
This study discovered the potential application of kaolin $(\mathrm{Kln})$ and silver-kaolin $(\mathrm{Ag}-\mathrm{Kln})$ in gelatin-composite films as active packaging material for food products. Three different types of kaolins; raw $\mathrm{Kln}, \mathrm{Ag}-\mathrm{K} \ln (1: 2) \mathrm{mix}$ and $\mathrm{Ag}-\mathrm{K} \ln (1: 1)$ mix with various concentrations (15\%, $30 \%$ and $45 \%$ ) were prepared by solution casting. For the water barrier properties, the wettability test indicated that the addition of kaolin in gelatin films produced hydrophobic films and lower $(\mathrm{p}<0.05)$ water vapour permeability, regardless of the kaolin type and concentration. Scanning electron microscopy images portrayed that higher inclusions of Ag-Kln compounds are able to develop smoother surface and homogenous cross-section. In addition, by incorporating these two materials, films with great antimicrobial effect towards both Grampositive and Gram-negative bacteria were produced. Elevation of Ag-Kln concentration also proved to lower the transmission of ultraviolet-vis light through the films. These findings suggested that gelatin film with $\mathrm{Ag}-\mathrm{K} \ln$ has a potential and beneficial in food packaging industry due to its enhanced properties. tilizer formulations for crop growth on acid soils.
\end{abstract}

Keyword: Biodegradable films; Active packaging; Fish gelatin; Kaolin; Silver 